\title{
A Decoupled Local Memory Allocator
}

\author{
BOUBACAR DIOUF, INRIA \\ CAN HANTAŞ, Georgia Institute of Technology \\ ALBERT COHEN, INRIA and École Normale Supérieure de Paris \\ ÖZCAN ÖZTURK, Bilkent University \\ JENS PALSBERG, UCLA
}

\begin{abstract}
Compilers use software-controlled local memories to provide fast, predictable, and power-efficient access to critical data. We show that the local memory allocation for straight-line, or linearized programs is equivalent to a weighted interval-graph coloring problem. This problem is new when allowing a color interval to "wrap around," and we call it the submarine-building problem. This graph-theoretical decision problem differs slightly from the classical ship-building problem, and exhibits very interesting and unusual complexity properties. We demonstrate that the submarine-building problem is NP-complete, while it is solvable in linear time for not-so-proper interval graphs, an extension of the the class of proper interval graphs. We propose a clustering heuristic to approximate any interval graph into a not-so-proper interval graph, decoupling spill code generation from local memory assignment. We apply this heuristic to a large number of randomly generated interval graphs reproducing the statistical features of standard local memory allocation benchmarks, comparing with state-of-the-art heuristics.
\end{abstract}

Categories and Subject Descriptors: D.3.4 [Programming Languages]: Processor-Compilers, Optimization

General Terms: Algorithms, Performance

Additional Key Words and Phrases: Local memory, scratchpad memory, memory allocation, compiler

\section{ACM Reference Format:}

Diouf, B., Hantaş, C., Cohen, A., Özturk, Ö., and Palsberg, J. 2013. A decoupled local memory allocator. ACM Trans. Architec. Code Optim. 9, 4, Article 34 (January 2013), 22 pages.

DOI $=10.1145 / 2400682.2400693$ http://doi.acm.org/10.1145/2400682.2400693

\section{INTRODUCTION}

Compilers use software-controlled local memories to provide fast, predictable, and power-efficient access to critical data. Predictability to data access and power consumption efficiency are often essential to real-time and embedded applications. Most ARM processors have an on-chip local memory [ARM 1998], and more generally, it is typical for DSPs and embedded processors to have local memories, also called scratchpad memories [Motorola 1998; Instruments 1997]. More specialized processors also utilize local memories, including stream-processing architectures such as graphical processors (GPUs) and network processors [NVIDIA 2008; Burns et al. 2003]. Most processor(s) may directly access the main memory — typically off-chip DRAM—resources,

This work is supported by the European Commission through the FP7 project TERAFLUX id. 249013.

Authors' addresses: B. Diouf (corresponding author), INRIA, France; email: boubacar.diouf@inria.fr; C. Hantas, Georgia Institute of Technology; A. Cohen, INRIA and École Normale Supérieure de Paris, France; O. Ozturk, Bilkent University, Turkey; J. Palsberg, UCLA.

Permission to make digital or hard copies of part or all of this work for personal or classroom use is granted without fee provided that copies are not made or distributed for profit or commercial advantage and that copies show this notice on the first page or initial screen of a display along with the full citation. Copyrights for components of this work owned by others than ACM must be honored. Abstracting with credit is permitted. To copy otherwise, to republish, to post on servers, to redistribute to lists, or to use any component of this work in other works requires prior specific permission and/or a fee. Permissions may be requested from Publications Dept., ACM, Inc., 2 Penn Plaza, Suite 701, New York, NY 10121-0701 USA, fax +1 (212) 869-0481, or permissions@acm.org.

(c) 2013 ACM 1544-3566/2013/01-ART34 $\$ 15.00$

DOI 10.1145/2400682.2400693 http://doi.acm.org/10.1145/2400682.2400693 
but few exceptions exist. The IBM Cell broadband engine's synergistic processing units (SPU) [Kahle et al. 2005] rely exclusively on DMA for instruction and data transfers with main memory. Our approach is compatible with such memory models.

In systems with local memories, data transfers between the main memory and the local memory are inserted into the generated code by the compiler or the application. Previous studies addressed local memory management from different angles, targeting for both application/code and data. These efforts considered both static [Avissar et al. 2002; Sjödin and von Platen 2001; Steinke et al. 2002] and dynamic methods [Udayakumaran and Barua 2003; Li et al. 2009]. Static methods either place an array in the local memory or in the off-chip memory during the whole execution of a program. Dynamic methods place an array in the local memory at a certain moment and in the off-chip memory at a different moment depending on its access frequency. Dynamic methods take into account the dynamic behavior of the program.

In this article, we consider an approach to local memory management that decouples spill code generation from local memory assignment. We show that the local memory allocation for straight-line programs or linearized programs, where the live ranges of variables or arrays are represented as intervals, is equivalent to a weighted intervalgraph coloring problem that we call the submarine-building problem. The submarinebuilding problem differs slightly from the classical ship-building problem [Golumbic 2004] by allowing a color interval to "wrap around." We show that the submarinebuilding problem is NP-complete, while it is solvable in linear time for not-so-proper interval graphs, an extension of the the class of proper interval graphs. We propose a novel approach to approximate any interval graph into a not-so-proper interval graph, decoupling spill code generation from local memory assignment. We apply this heuristic to a large number of randomly generated weighted interval graphs reproducing the statistical features of standard local memory allocation benchmarks. We compare our approach with state-of-the-art heuristics.

\section{MOTIVATION}

In a previous paper [Diouf et al. 2009], based on recent progress in register allocation, we considered a decoupled approach to local memory allocation, and we experimentally validated this decoupling. This article takes a more theoretical standpoint and seeks to better understand the optimization problem of local memory management.

Recent research in register allocation leverages the complexity and performance benefits of decoupling its allocation and assignment phases [Appel and George 2001; Pereira and Palsberg 2005; Hack et al. 2005; Bouchez et al. 2006b; Brisk et al. 2006]. The allocation phase decides which variables to spill and which to assign to registers. The assignment phase chooses which variable to assign to which register.

The allocation phase relies on the maximal number of simultaneously living subvariables, called MAxLive, a measure of register pressure. When enough live range splitting is done, it is sufficient that MAXLIVE is less or equal to the number of available registers to guarantee that all the subvariables will be allocated and the forthcoming assignment phase can be done without further spill. In many cases, assignment can even be achieved in linear time [Hack et al. 2006; Bouchez et al. 2006b]. If at some program point the pressure exceeds the number of available registers, MAXLIVE needs to be reduced through spilling.

This decoupled approach permits to focus on the hard problem, namely the spilling decisions. It also improves the understanding of the interplay between live range splitting and the expressiveness and complexity of register allocation. This is best illustrated by the success of SSA-based allocation [Bouchez et al. 2006a, 2006b, 2007; Hack et al. 2006; Braun and Hack 2009]. 
The intuition for decoupled register allocation derives from the observation that live range splitting is almost always profitable if it allows to reduce the number of register spills, even at the cost of extra register moves. The decoupled approach focuses on spill minimization only, pushing the minimization of register moves to a later register coalescing phase [Appel and George 2001; Hack et al. 2006; Bouchez et al. 2008]. Here again, SSA-based techniques have won the game. Specifically, they collapse the register coalescing with the hard problem of getting out of SSA [Hack et al. 2006; Boissinot et al. 2009; Pereira and Palsberg 2009], as one of the last backend compiler passes.

The domain of local memory management tells a very different story. Some heuristics exist [Udayakumaran and Barua 2003; Udayakumaran et al. 2006; Kandemir et al. 2001; Li et al. 2009] but little is known about the optimization problem, its complexity, and the interplay with other optimizations. The burning hot question is of course: does the decoupled approach hold for the local memory management problem? Surprisingly, the state-of-the-art of local memory management completely underexploits all the advances in register allocation. When focusing on arrays, the similarity between register and local memory allocation is obvious nonetheless.

-Local memory allocation. Deciding which array blocks to spill to main memory and which array blocks to allocate to the local memory. Spilling is typically supported by DMA units.

-Local memory assignment. Deciding at which local memory offset to assign which allocated array block.

In the context of local memory management, the maximum size of simultaneously living arrays, ${ }^{1}$ called MaxSize, gives a measure of local memory pressure. Again, like for register allocation, live range splitting helps to reduce the local memory pressure. Since arrays are frequently accessed inside loops, local memory management algorithms often split arrays at loop-entry points; we call these points decision points. Decision points can also be chosen in a finer manner, after loops or before array accesses. Local memory pressure can also be reduced by loop transformations like strip-mining, tiling which reduces the portion of accessed arrays. For all these reasons, the study of a decoupled approach in the local memory management context seems very appealing.

\section{FROM LOCAL MEMORY MANAGEMENT TO WEIGHTED GRAPH COLORING}

This section sets the terminology and definitions used in the rest of the article.

\subsection{Weighted Graphs}

A graph $G=(V, E)$ consists of two sets, $V$ the set of vertices, and $E$ the set of edges. Every edge $\left(v_{1}, v_{2}\right)$ of $E$ has two end points $v_{1} \in V$ and $v_{2} \in V$. We consider undirected graphs only, i.e., we do not make difference between the edges $\left(v_{1}, v_{2}\right)$ and $\left(v_{2}, v_{1}\right)$.

A graph $G$ is called an interval graph if its vertices can be put into one-to-one correspondence with a set of intervals $I$ of a linearly ordered set such that two vertices are connected by an edge of $G$ if and only if their corresponding intervals have a nonempty intersection.

Assuming each vertex $v$ of $G=(V, E)$ is associated with a nonnegative number $w(v)$, the weight of a subset $S \subset V$ is expressed as

$$
w(S)=\sum_{v \in S} w(v)
$$

The graph $G$ associated with the function $w$ is called a weighted graph and denoted $G_{w}$. Moreover, $G_{w}$ is a weighted interval graph if $G$ is an interval graph.

\footnotetext{
${ }^{1}$ Not the number of simultaneously living arrays.
} 


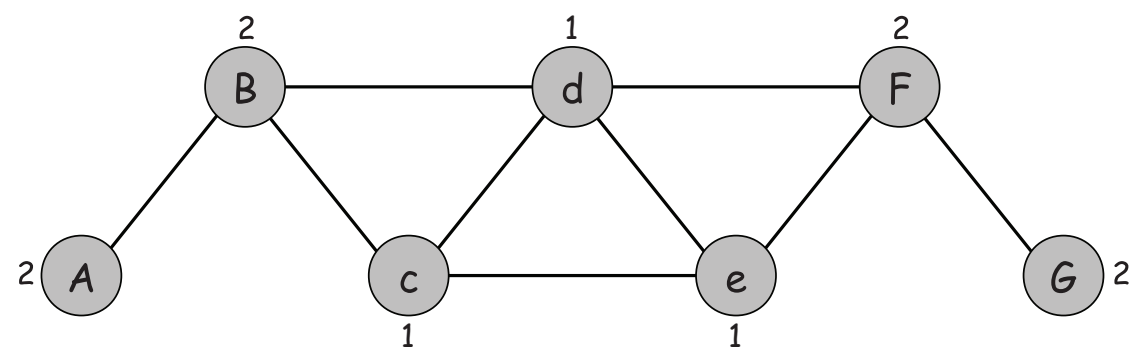

(a)

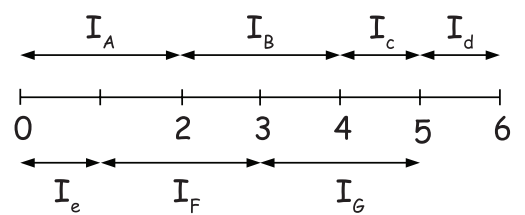

(b)

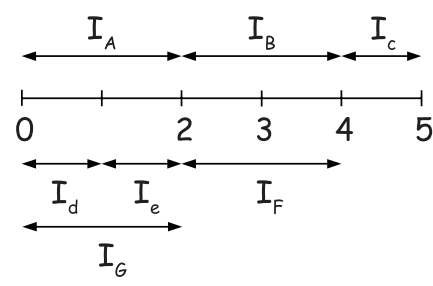

(c)

Fig. 1. Two colorings of a weighted graph.

An interval coloring of a weighted graph $G_{w}$ is a function $I$ mapping each vertex $v \in V$ onto an (open) interval $I_{v}$ of $w(v)+1$ consecutive integers of the real line, such that adjacent vertices are mapped to disjoint intervals; that is, $\left(v_{1}, v_{2}\right) \in E$ implies $I_{v_{1}} \cap I_{v_{2}}=\emptyset$. We say that $I$ is a $k$-coloring of $G_{w}$ if $I_{v} \in\{0, \ldots, k\}, \forall v \in V$. The chromatic number $\chi\left(G_{w}\right)$ is the smallest $k$ for which we can find a $k$-coloring of $G_{w}$.

Figure 1(b) and Figure 1(c) show two colorings of the weighted graph shown in Figure 1(a). Figure 1(b) presents a 6-coloring of the weighted graph and Figure 1(c) shows a 5-coloring of the weighted graph. The chromatic number of this graph is 5 .

\subsection{Straight-Line Programs and Linearized Programs}

Given an intermediate representation of an arbitrary program, the intermediate representation pseudo-instructions can be numbered according to some order. We define a linearized program as a program for which such kind of numbering has been performed and for each variable $v$ in this program, we represent its live range as the live interval $[i, j[, i$ being the number of the first instruction where $v$ is first defined and $j$ being the number of the instruction where $v$ is last used. There can be some pseudo-instructions between $i$ and $j$ where $v$ is not live, but with a successful live range splitting this problem can become marginal. In the context of just-in-time compilation where compilation is critical, linearizing programs can pay off because it is fast to linearize a program and hopefully the produced code could be of relative good quality [Sarkar and Barik 2007].

\subsection{Two Sides of the Coin}

We demonstrate the equivalence between allocating the local memory for a linearized program and coloring a weighted interval graph.

From linearized programs to weighted interval graphs. From a linearized program we construct a corresponding weighted graph called interference graph. For each variable 
in this program, we create a vertex and associate the size of the variable to this vertex. We create an edge between two vertices if there is a point in the program where the two variables are simultaneously live. Thus, an edge connects a pair of vertices if and only if the variables are simultaneously live. The constructed weighted graph is a weighted interval graph because each vertex corresponds to an interval defined by the definition point and the end point of the variable.

From weighted interval graphs to linearized programs. We use a method similar to the one presented by Lee et al. [2008] to show that, for any weighted interval graph, we can exhibit a corresponding linearized program.

Chen [1992] and Saha et al. [2007] have shown how to convert an interval graph with $q$ intervals to an isomorphic program-like interval graph in $\mathcal{O}(q \log q)$ time. An interval graph is program-like if the intervals representing the vertices of the graph have start points and end points that are all different, and the start points and end points of the intervals form a set $\{1, \ldots, 2 q\}$, where $q$ is the number of intervals.

From a program-like weighted interval graph $G_{w}$, we construct in $\mathcal{O}(q)$ time the following straight-line program (with pseudo-C syntax) which consists of a set of $2 q$ statements.

$$
\forall i \in\{1, \ldots, 2 q\}\left\{\begin{array}{c}
\text { type }_{I} \mathrm{v}_{I}=\cdots, \\
\text { where sizeof }\left(\text { type }_{I}\right)=w(I), \\
\text { if the interval } I \text { of weight } w(I) \text { begins at } i \\
\cdots=\mathrm{v}_{I}, \\
\text { if the interval } I \text { ends at } i .
\end{array}\right.
$$

\section{WEIGHTED GRAPH COLORING}

Thirty years ago, in her seminal paper [Fabri 1979], Fabri already envisaged to model the so-called problem of "automatic storage allocation" as a weighted graph coloring problem. She mentions the investigation of special subclasses of weighted graphs that are likely to occur. We construct a weighted graph $G_{w}$ from a given linearized program. Finding an allocation for variables of the linearized program within a local memory of size $k$ corresponds to finding a $k$-coloring of $G_{w}$.

This section introduces the ship-building problem which is related to weighted interval graph coloring. It also defines a new variant of the ship-building problem, called the submarine-building problem, very well suited to the local memory allocation problems on modern processors, and exhibiting interesting complexity results and approximation heuristics.

\subsection{The Ship-Building Problem}

We report here the ship-building problem as presented in the book of Golumbic [2004]. In certain shipyards the sections of a ship are constructed on a dry dock, called the welding plane, according to a rigid time schedule. Each section $s$ requires a certain width $w(s)$ on the dock during construction. Can the sections be assigned space on a welding plane of total width $k$ so that no spot is reserved for two sections at the same time?

Let the sections be represented by the vertices of a graph $G$ and connect two vertices if their corresponding sections have intersecting time intervals. Thus $G_{w}$ is a weighted interval graph. An interval coloring of $G_{w}$ will provide the assignment of the sections 


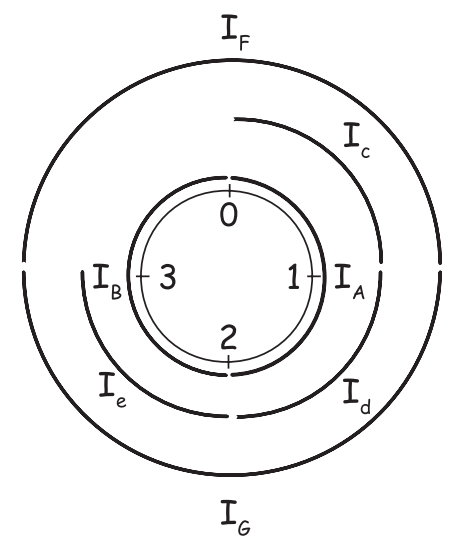

Fig. 2. An example of a 4-submarine-coloring.

to spaces, of appropriate size, on the welding plane. This assignment will be consistent with the intersecting time restrictions. The reader must be careful to distinguish between the time intervals which produced the edges of $G_{w}$ and the color intervals which provide a solution to the assignment of space on the dock.

A weighted graph built from a linearized program associated with a number $k$ (corresponding to the size of the local memory) is an instance of the ship-building problem. For a graph $G_{w}$ and a number $k$, we call $\operatorname{ship}\left(G_{w}, k\right)$ an instance of the ship-building problem.

Determining whether $\chi\left(G_{w}\right) \leq k$ is an NP-complete problem [Golumbic 2004; Lee et al. 2008], ${ }^{2}$ even if $G$ is an interval graph and the weight function $w$ is restricted to the values 1 and 2 . It follows that the ship-building problem is also NP-complete.

\subsection{The Submarine-Building Problem}

Since the local memory size is generally power-of-two, it is common to mask the addresses (in software or hardware) to let loads and stores wrap around to the local memory transparently. The submarine-building problem is a new variant of the shipbuilding problem. Like in the ship-building problem, a vertex must occupy a contiguous color interval, but a circular allocation scheme can be adopted permitting a color interval to wrap around. It extends the ship-building problem's interval coloring to circular interval coloring. It follows that a solution of the ship-building problem is a solution to the submarine-building problem, but the converse is not generally true. Figure 2 shows an example of submarine coloring for the weighted graph in Figure 1(a). The inner circle represents the colors and each circular arc $I_{v}$ represents a color interval assigned to the vertex $v$. The color interval $I_{F}$ wraps around.

For a weighted graph $G_{w}$ and a number $k$, we call submarine $\left(G_{w}, k\right)$ an instance of the submarine-building problem. For the rest of the article we say that $G_{w}$ is $k$-shipcolorable, if $\operatorname{ship}(G, k)$ has a solution, and we also say that $G_{w}$ is $k$-submarine-colorable, if submarine $\left(G_{w}, k\right)$ has a solution.

To the best of our knowledge, this variant of the ship-building problem has never been carefully studied, and it has not been applied to the decoupling of the spilling and assignment problems in local memory management. Many open questions about

\footnotetext{
${ }^{2}$ This has been previously proved by Stockmeyer, but to the best of our knowledge, the proof of Lee et al. is the first publicly available one [Lee et al. 2008].
} 
fragmentation, optimality, complexity, and feasibility are tied to this new variant of the ship-building problem.

Unfortunately, the submarine-building problem is also NP-complete on weighted interval graphs as we demonstrate next.

\section{Theorem 4.1. The submarine-building problem is NP-complete.}

Proof. To show that the submarine-building problem is NP-complete, we first show that it is a problem in NP, and we then show how to build from an instance $\operatorname{ship}(G, k)$ of the ship-building problem an instance submarine $\left(G_{w}, k+1\right)$ of the submarine-building problem.

A problem in NP. The submarine-building problem is in NP because a solution of the problem can be verified polynomially.

Reduction. From an instance $\operatorname{ship}\left(G_{w}, k\right)$ of the ship-building problem, we build an instance submarine $\left(G_{w}, k+1\right)$ of the submarine-building problem. Let $f$ and $\ell$ be respectively the minimum of the start points of all intervals in $G_{w}$ and the maximum of the end points of all intervals in $G_{w}$. The graph $G_{w}^{\prime}$ consists of all intervals of $G_{w}$ and the interval $\beta$ : [ $f, \ell[$ of weight one.

Let $\theta$ be a solution of $\operatorname{ship}\left(G_{w}, k\right) ; \theta$ maps each interval of $G_{w}$ to a color interval between 0 and $k$. We define $\theta^{\prime}$, a function mapping each interval $\alpha$ of $G_{w}^{\prime}$ to a color interval between 0 and $k+1$.

$$
\forall \alpha \in G_{w}^{\prime} \begin{cases}\theta^{\prime}(\alpha)=\theta(\alpha) & \text { if } \alpha \in G_{w} \\ \theta^{\prime}(\alpha)=[k, k+1[ & \text { if } \alpha \notin G_{w}\end{cases}
$$

It follows that $\theta^{\prime}$ is a solution of submarine $\left(G_{w}, k+1\right)$.

Now, we study the converse case. Let $\theta^{\prime}$ be a solution of $\operatorname{submarine}\left(G_{w}, k+1\right)$. We define for a color interval $[s, e[$, an integer $k$, and the functions $\delta$ and $\bmod$.

$$
\begin{aligned}
\delta([s, e[, d) & =[s+d, e+d[ \\
\bmod ([s, e[) & =[s \bmod (k+1), e \bmod (k+1)[
\end{aligned}
$$

Let $\theta^{\prime}(\beta)=\left[s, s+1\left[\right.\right.$ ( $\beta$ is of weight one). We define for each interval $\alpha$ of $G_{w}$ the function $\theta$.

$$
\theta(\alpha)=\bmod \left(\delta\left(\theta^{\prime}(\alpha), k-s\right)\right)
$$

The interval $\beta$ of $G_{w}^{\prime}$ is live from $f$ to $\ell$, therefore there is no other interval of $G_{w}^{\prime}$ that occupies the color interval $[s, s+1[. \theta([s, s+1[)=[k, k+1$ [, and the value on which function $\theta$ is equal to [k,k+1[ is [s,s+1[. Thus, $\theta$ assigns to each interval $\alpha$ of $G_{w}$ an interval of some color between 0 and $k$. If two interfering intervals $\alpha$ and $\alpha^{\prime}$ have two nonoverlapping color intervals $c$ and $c^{\prime}$ then $\theta(\alpha)$ and $\theta\left(\alpha^{\prime}\right)$ are nonoverlapping too. It follows that $\theta$ is a solution of $\operatorname{ship}\left(G_{w}, k\right)$ if $\theta^{\prime}$ is a solution of the $\operatorname{submarine}\left(G_{w}, k+1\right)$.

\section{WEIGHTED PROPER INTERVAL GRAPH COLORING}

We study the properties of weighted proper interval graphs, a subclass of weighted interval graphs. This class is interesting because we will show the submarine-building problem is solvable in linear time for this class: any instance $G_{w}$ of this class is colorable with $\omega\left(G_{w}\right)$ colors and in linear time. For this subclass, we also have a sufficient criterion permitting to decouple the ship-building problem.

\subsection{Proper Interval Graph}

An interval graph $G$ is a proper interval graph if it is constructed from a family of intervals such that no interval properly contains another [Golumbic 2004]. An interval 


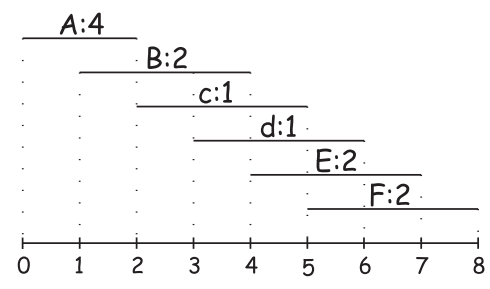

(a)

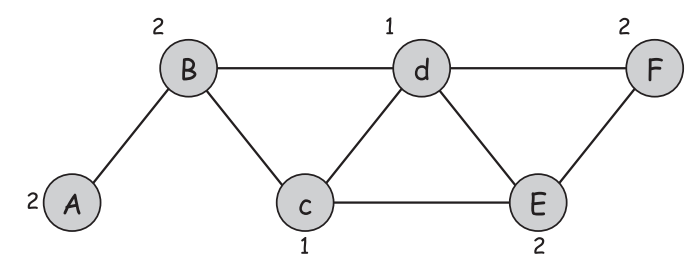

(b)

Fig. 3. An example of a weighted proper interval graph.

graph is a unit interval graph if all of its intervals have the same length. It has been shown that the classes of proper interval graphs and the unit interval graphs coincide. A weighted graph $G_{w}$ is a weighted proper interval graph if $G$ is a proper interval graph. Figure 3 shows properly ordered weighted intervals of the real line and their corresponding weighted proper interval graph.

\subsection{Proper Ordering}

Let us consider the representation of $G_{w}$, a weighted proper interval graph, on the real line, where the vertices of $G_{w}$ correspond to intervals on the real line. Let us sort these intervals according to their start points. If two intervals $i$ and $i^{\prime}$ start at the same point, we can place either $i$ before $i^{\prime}$ or $i^{\prime}$ before $i$. This kind of ordering can be found for any weighted proper interval graph, and is called proper ordering in our approach. A proper ordering of the graph in Figure 3 is: $A, B, c, d, E, F$. Based on this ordering, we say that $i \prec i^{\prime}$, if $i$ is before $i^{\prime}$.

LEMMA 5.1. If $i \prec i^{\prime}$ then, either $i$ ends before $i^{\prime}$ or $i$ and $i^{\prime}$ start and finish at the same time.

Proof. $i \prec i^{\prime}$ implies that either $i$ starts before $i^{\prime}$ or $i$ and $i^{\prime}$ starts at the same time:

$-i$ starts before $i^{\prime}$. Since $i$ cannot properly contain $i^{\prime}$, then $i$ ends before $i^{\prime}$.

$-i$ and $i^{\prime}$ start at the same time. Since, none of these two intervals cannot properly contain the other, then they end at the same time.

\subsection{Decoupled Submarine-Building Problem}

Algorithm 1 performs a $k$-submarine-coloring of intervals of a weighted proper interval graph $G_{w}$. It takes as input a sequence of intervals of $G_{w}$ sorted according to a proper ordering. It assigns to every interval a color interval contiguous to the color interval assigned to the previous interval (according to the proper ordering) in a clockwise manner. Finally, it gives a $k$-submarine-coloring of the graph as output.

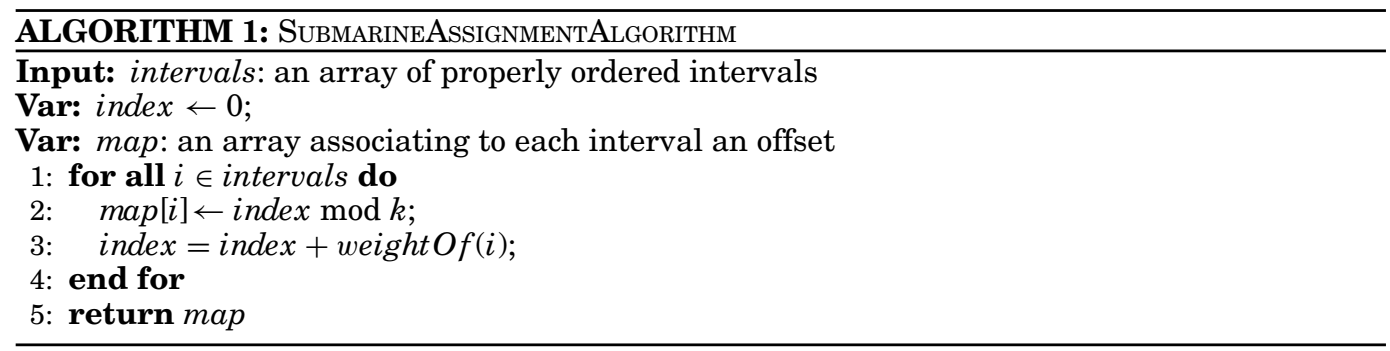


Theorem 5.2. For any weighted proper interval graph $G_{w}$, Algorithm 1 guarantees a $k$-submarine-coloring if and only if $\omega\left(G_{w}\right) \leq k$.

Proof.

Direct. $k$-submarine-coloring of $G_{w} \Longrightarrow \omega\left(G_{w}\right) \leq k$.

Any $k$-submarine-coloring of $G_{w}$ must assign color intervals that do not overlap to the intervals of a clique of weight $\omega\left(G_{w}\right)$ and this is only possible if $\omega\left(G_{w}\right) \leq k$.

Reciprocal. $\omega\left(G_{w}\right) \leq k \Longrightarrow k$-submarine-coloring of $G_{w}$.

We will call a point, the moment an interval starts. Let $P$ be the following property: "at point $n$, the live intervals $i_{j}, i_{j+1}, \ldots, i_{n}$ (sorted according to the proper ordering) are assigned to contiguous color intervals that do not overlap in a clockwise manner, in this order: $\operatorname{color}\left(i_{j}\right), \operatorname{color}\left(i_{j+1}\right), \ldots \operatorname{color}\left(i_{n}\right)$ ". The property $P$ is an invariant at every point of Algorithm 1. If the graph contains $m$ nodes, we have consequently $m$ intervals and $m$ points. The proof will be done inductively on points.

Just before the point 1 , where the first interval $i_{1}$ starts, none of the color intervals is used. At point 1 , Algorithm 1 assigns to $i_{1}$ a color interval starting at 0 and property $P$ is trivially satisfied.

Suppose that property $P$ is satisfied from point 1 to point $n$, and let us see if property $P$ is satisfied at point $n+1$ (we assume that we have at least $n+1$ intervals in the graph). We call $d$ the number of dead intervals between $n$ and $n+1$ ( $d$ can be zero, or $n-j$ ), we prove four claims successively:

(1) $i_{j+d}$ is live. Indeed, if $i_{j+d}$ was dead then all intervals preceding it would also be dead. Therefore, we would have $d+1$ intervals that are dead, which contradicts the definition of $d$.

(2) All the intervals between $i_{j+d}$ and $i_{n}$ are live too. If an interval $i_{k}$ between $i_{j+d}$ and $i_{n}$ is dead then $i_{j+d}$ is also dead because $i_{j+d} \prec i_{k}$; this leads to a contradiction with the first claim.

(3) From the two first claims and the satisfaction of proposition $P$ at point $n$, we deduce that all live intervals $i_{j+d}, i_{j+d+1}, \ldots, i_{n}$ are assigned to contiguous colors that do not overlap, in a clockwise manner, in this order: $\operatorname{color}\left(i_{j+d}\right), \operatorname{color}\left(i_{j+d+1}\right), \ldots \operatorname{color}\left(i_{n}\right)$.

(4) Algorithm 1 assigns to the new interval $i_{n+1}$ a color interval contiguous to the last used color interval (the color of $i_{n}$ ) in a clockwise manner. Therefore, the color intervals assigned to live intervals are contiguous in a clockwise manner, in this order: $\operatorname{color}\left(i_{j+d}\right), \operatorname{color}\left(i_{j+d+1}\right), \ldots$ color $\left(i_{n}\right), \operatorname{color}\left(i_{n+1}\right)$. The colors do not overlap because they are all contiguous and they do not exceed $\omega\left(G_{w}\right)$ which does not exceed $k$.

From the fourth claim, we conclude that at the point $n+1$ the property $P$ is again verified.

Hence, using Algorithm 1 guarantees that at every point, all the live intervals are assigned to contiguous color intervals that do not overlap, and the next starting interval will be assigned to a color interval. Thus, a $k$-submarine-coloring can be found for $G_{w}$ if $\omega\left(G_{w}\right) \leq k$.

As far as we are aware, this is the first time a decision problem is shown to be NPcomplete on interval graphs and polynomial on unit interval graphs (which are equivalent to proper interval graphs).

\section{WEIGHTED NOT-SO-PROPER INTERVAL GRAPHS}

We say that two intervals $A$ and $B$ properly interfere if $A$ interferes with $B$ such that $A$ strictly starts before $B$ and $B$ strictly ends after $A$ or vice versa. 


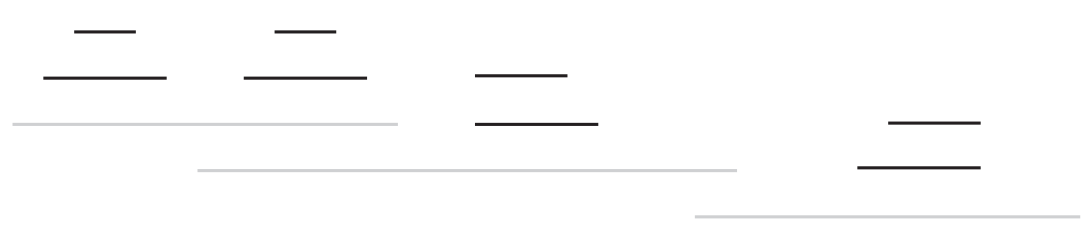

Fig. 4. An example of weighted NSP graph.

(a)

(b)

Fig. 5. Two graphs that are not weighted NSP graphs.

We define a weighted Not-So-Proper (NSP) interval graph as a weighted interval graph, where each pair of properly interfering intervals $A$ and $B$ is such that $A$ and $B$ must not be contained in any other interval of the graph.

Figure 4 shows an example of a weighted NSP graph (weights have been omitted in the figure), whereas Figure 5 illustrates two weighted graphs that are not NSP. The light gray lines represent intervals that are not contained in other intervals, the solid black lines represent intervals that are contained, and the black dashed lines represent the intervals we do not want to have in weighted NSP graphs.

The weighted NSP graphs are the class of graphs that includes the weighted proper interval graphs and the superperfect graphs defined by Li et al. [2011]. Thus, when the submarine assignment problem is considered, the weighted NSP interval graphs are guaranteed to be MaxSize-colorable.

Algorithm 2 performs a submarine assignment for a weighted NSP graph on a local memory of size MaxSize. It receives as input intervals, a list of intervals sorted by increasing start point, and returns at the end map, a map that associates to each interval an offset into the local memory. This algorithm differentiates between intervals that are not contained in any other interval, called containers and those contained in an interval. The contained intervals are stocked into stack. The variable container keeps track of the last starting container. When a new interval, $i$, starts, it is verified for containment-the function ConTAINS(container, $i$ ) returns true, if $\mathrm{i}$ is contained in container and false otherwise-and if it is not contained in the currently live intervals, it is set as the new container. Then, all the dead intervals are removed from stack and the offset is updated. The function $\operatorname{WeIGHTOF}(i)$ returns the weight of the interval $i$. If $i$ is contained into another interval, it is pushed onto stack. Finally, $i$ is assigned to the current offset, which is then updated.

\section{LOCAL MEMORY ALLOCATION THROUGH WEIGHTED NSP GRAPH COLORING}

As explained in Section 3, from a linearized program it is possible to construct a corresponding weighted interval graph. If the resulting graph is a weighted NSP interval graph, when the submarine assignment problem is considered, it is always possible to use MaxSize as a criterion to ensure that the assignment phase is feasible without spills. Thus, the allocation algorithm can be decoupled thanks to the MaxSize criterion. For arbitrary interval graphs, the problem is NP-complete and a heuristic-based solution should be envisaged.

We devised a solution that takes advantage of our submarine assignment algorithm. This solution that decouples the allocation and assignment, performs the two following steps: 

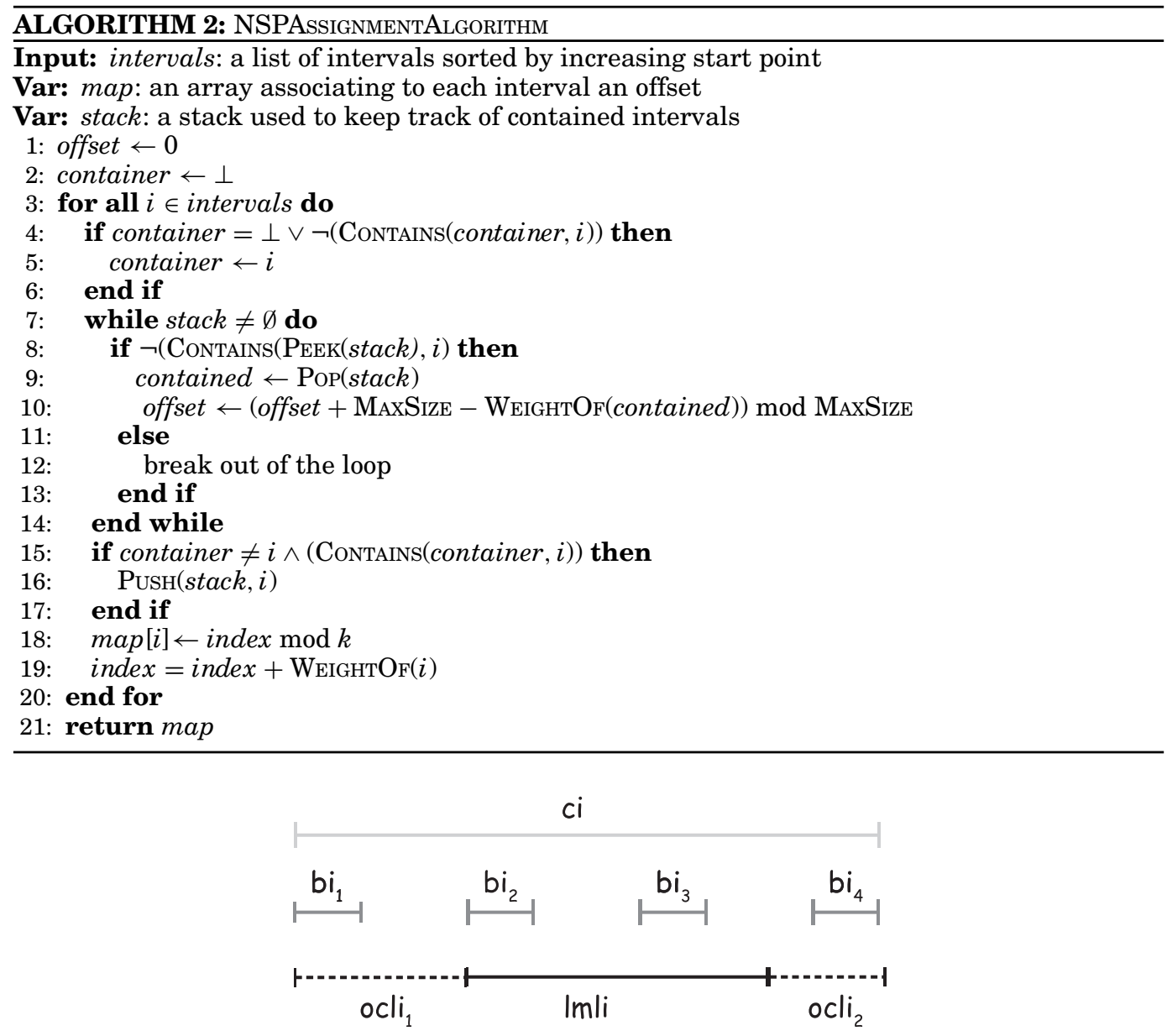

Fig. 6. An example of live intervals.

(1) it approximates an arbitrary weighted interval graph into a weighted NSP graph through spilling and splitting;

(2) it performs a submarine assignment with Algorithm 2.

\subsection{Live Ranges Representation}

Each array of a program is represented by a live interval (compound interval) starting from its first definition to its last use. Within the compound interval, there may exist some idle subintervals where the array is not accessed at all and other subintervals where the array is frequently accessed. The latter are called basic intervals. These basic intervals often correspond to loops, because arrays are generally frequently accessed through loops. Figure 6 shows an example of compound interval (ci) and the basic intervals $\left(b i_{1}, b i_{2}, b i_{3}, b i_{4}\right)$ that compose $c i$. Usually it is not recommended to split the live interval of an array inside a loop, because the incurred memory transfer cost will be too high, hence in our approach we do not split a compound interval inside one of its basic interval.

During the execution of a program, an array may occupy different locations in memory. The sublive range-a subinterval of the array's compound interval—during which 
the array occupies a specific location in memory, is called a memory live interval. A memory live interval is composed of a set of successive basic intervals of the compound interval. All the basic intervals in a memory live interval belong to the same compound interval. But, on the other hand, two basic intervals of the same compound interval may belong to different memory live intervals. If the array occupies a unique location in memory during all its compound interval, the memory live interval is the same as the compound interval. If the array is located in the local memory during the memory live interval, it is called a local-memory live interval. In contrast, if it is in the off-chip memory, it is called off-chip live interval. Figure 6 shows an example of configuration that can happen during execution. The array $A$ which has $c i$ as compound interval is in the local memory during the interval $l m l i$, a local-memory live interval. $A$ is in the off-chip memory during $o c l i_{1}$ and $o c l i_{2}$, two off-chip live intervals.

\subsection{The Approximation Algorithm}

Based on the notions of local-memory live interval and off-chip live interval, we devise an approximation algorithm which transforms a weighted interval graph into a weighted NSP interval graph composed of local-memory live intervals through spilling and splitting of the live intervals. This approximation is performed by Algorithm 3 .

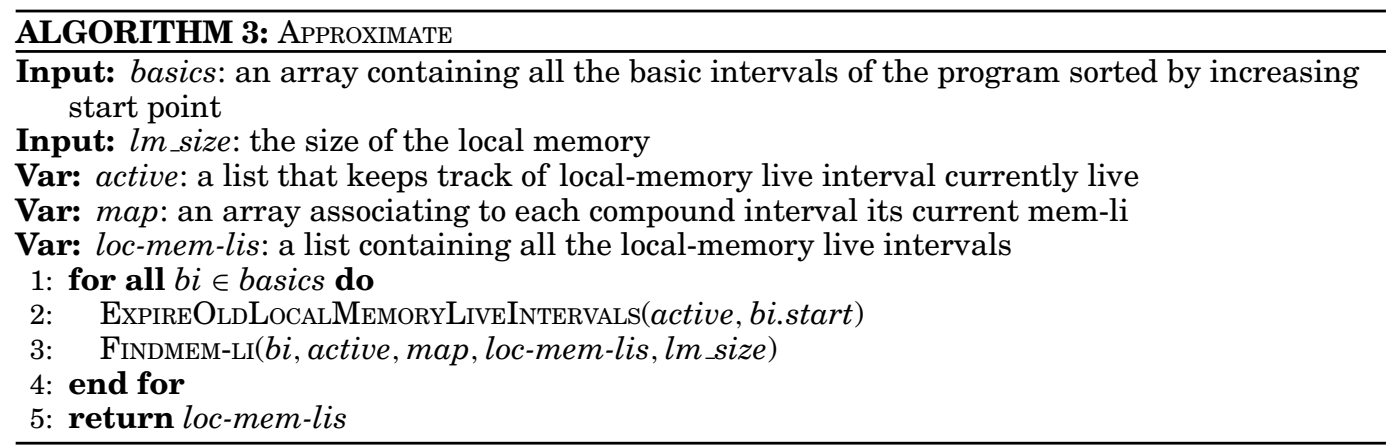

Algorithm 3 receives as input basics, an array containing all the basic intervals of the program sorted by increasing start point, and lm_size the size of the local memory. It then creates two variables, active and map. At every moment, active keeps track of the local-memory live intervals that are live and map associates to each compound interval its latest computed memory live interval. For each basic interval, $b i$, Algorithm 3 first removes from active the local-memory live intervals of a compound interval whose end point is lower than the start point of $b i$. Afterwards, Algorithm 3 attempts to add $b i$ into a local-memory live interval. If this is impossible $b i$ becomes part of an off-chip live interval. When all of the basic intervals of the program are processed, Algorithm 3 returns loc-mem-lis, the list of all the local-memory live intervals which are the intervals of the approximated weighted NSP interval graph.

The weighted NSP interval graph is computed iteratively with Algorithm 4. Algorithm 4 aims to assign bi's array to a location in the local memory based on the currently active local-memory live interval. It first retrieves the latest memory live interval of bi's compound interval ( bi.compound) into mem-li. Three different cases can happen.

(1) mem-li does not correspond to any value. It means that this is the first time the array of the compound interval is accessed. In this case Algorithm 4 creates new_mem-li, a temporary new local-memory live interval with $b i$, and checks, with the function AllocateOrNot(), if it can be added to active. If it is the case, new_mem-li is added 


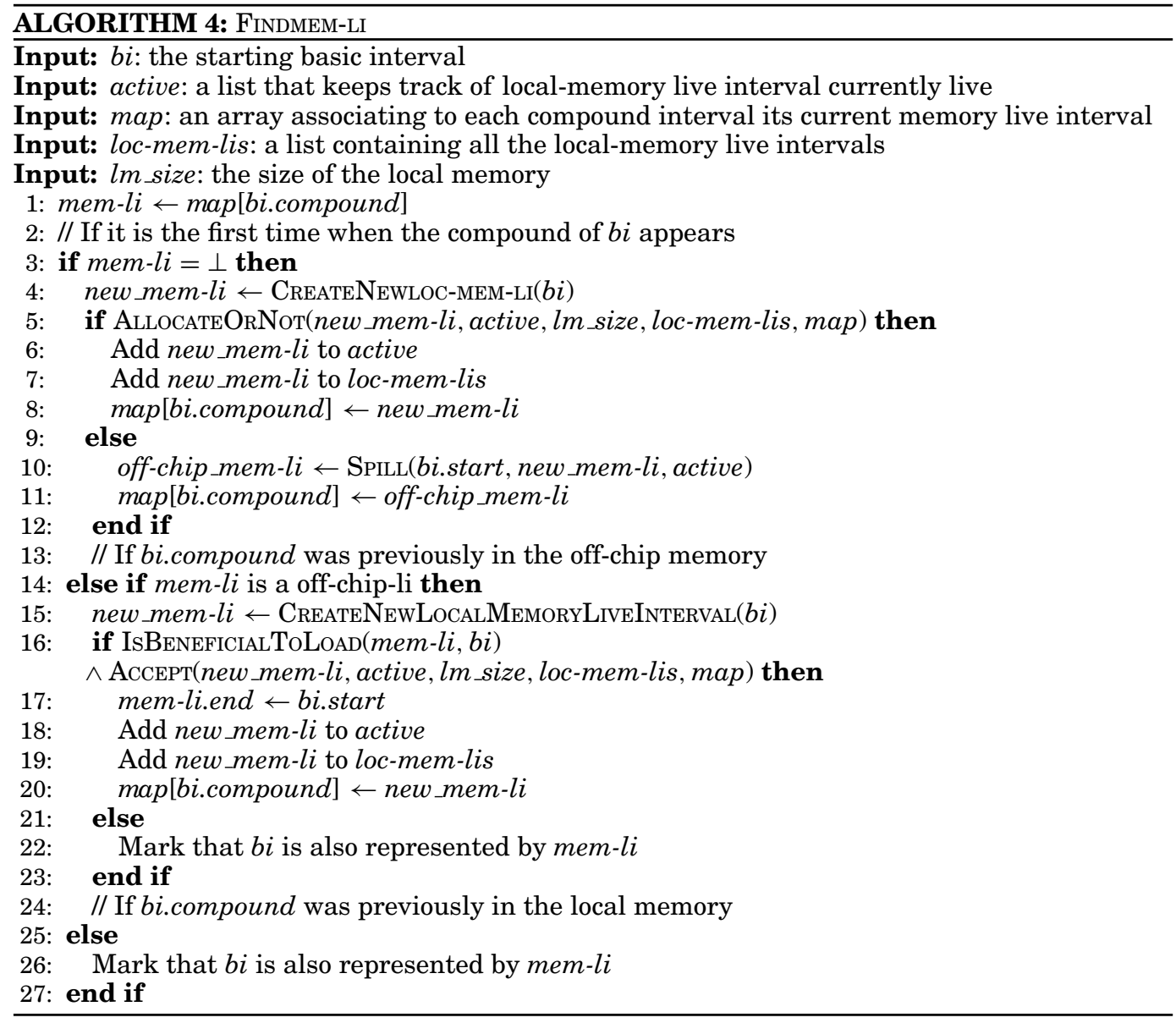

to active and to loc-mem-lis, and bi's compound is is associated to new mem-li. Otherwise, $b i$ will be part of off-chip_mem-li, an off-chip live interval, and $b i$ 's compound is associated to off-chip mem-li.

(2) mem-li corresponds to an off-chip live interval. If the cost of loading bi's array is lower than the cost of accessing it from the off-chip memory-the function IsBeneficialToLoAD () returns true-and there is enough room for it-the function AllocateOrNot() returns true-then mem-li's end point is set to bi's start point, new_mem-li is added to active and to loc-mem-lis, and bi's compound is associated to new_mem-li. Otherwise, $b i$ will be part of mem-li, which is an off-chip live interval in this case.

(3) mem-li corresponds to a local-memory live interval. In this case, Algorithm 4 marks that $b i$ will be part of mem-li, which is a local-memory live interval in this case.

The function AllocateOrNot() used in Algorithm 4 is described by Algorithm 5. This algorithm spills or splits either the active local-memory live intervals or spills new_mem-li which corresponds to transforming it into an off-chip live interval. When Algorithm 5 is invoked, it checks if the sum of the weights of new_mem-li and the localmemory live intervals currently in active — given by the function WeIGHTOF()—is lower 


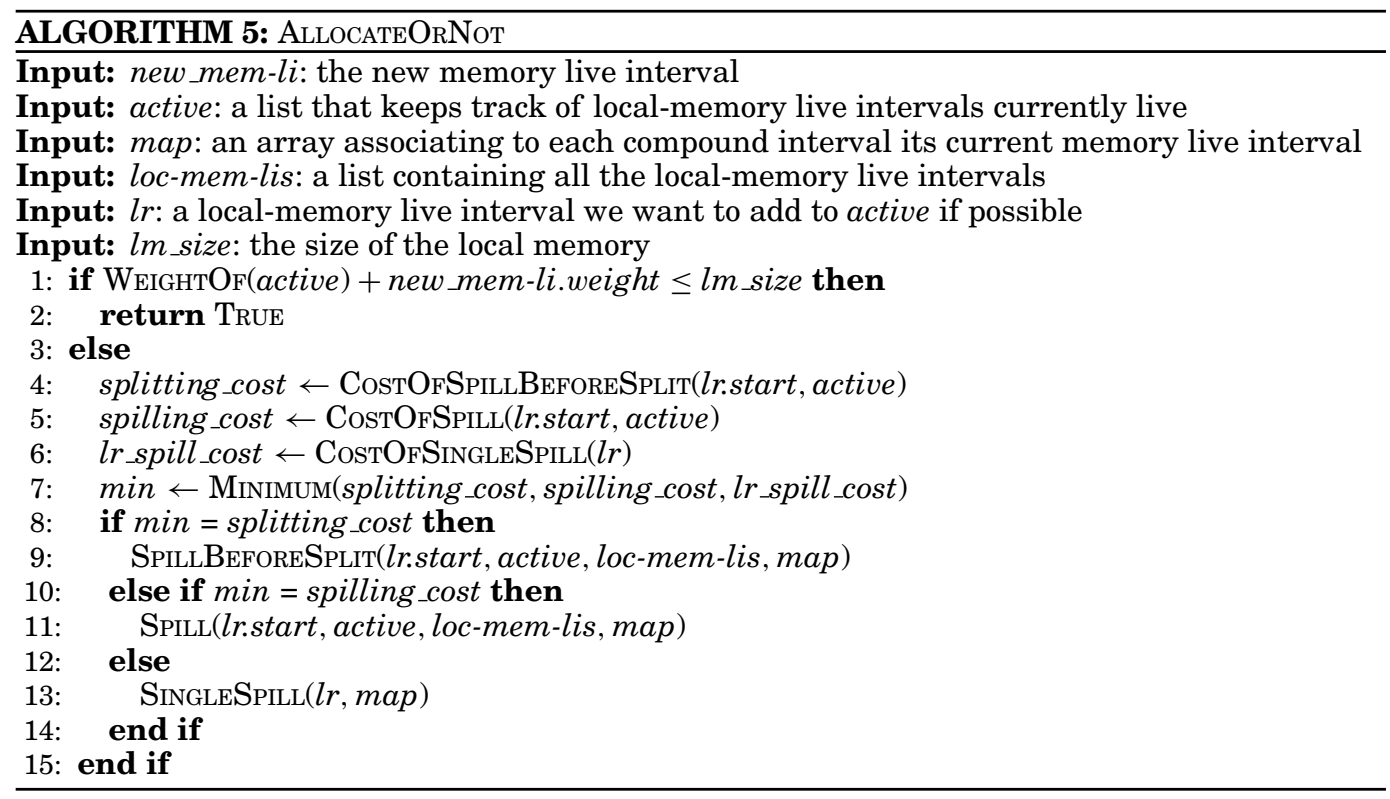

than $l m$ size, the size of the local memory. In this case, it returns true to report that new_mem-li can be added to active. Otherwise, Algorithm 5 considers three possibilities.

(1) First, it computes the cost of splitting the local-memory live intervals when new_mem-li starts. Note that the local-memory live intervals, currently used at that moment, which have a basic interval that contains the start point of new_mem-li, could not be split at that moment. Algorithm 5 computes the cost of spilling these local-memory live intervals first, then if some space is still needed to hold new_mem$l i$, it computes the cost of breaking some local-memory live interval into a localmemory live interval which stops now, at the moment when new mem-li starts, and an off-chip live interval from now. It breaks the local-memory live intervals until new_mem-li can fit.

(2) It computes the cost of spilling some local-memory live intervals until new_mem$l i$ can fit. Algorithm 5 walks over the local-memory live intervals in the order of increasing spill cost.

(3) It computes the cost of spilling new_mem-li.

Algorithm 5 selects the possibility that is the least expensive and updates accordingly the variables map, active, and loc-mem-lis.

\section{EXPERIMENTAL EVALUATION}

To evaluate our approach we have generated 2000 graphs (1000 superperfect graphs and 1000 arbitrary interval graphs) with various properties. We compared our approach with the polynomial allocator of $\mathrm{Li}$ et al. [2009] which is the closest work to ours. We also compare our approach with the classical best-fit allocator and to a variant of the best-fit which aims to reduce copies. Notice both best-fit algorithms are exponential in the size of the local memory: they are very effective for a few hundreds of kilobytes, but they may not scale to larger allocation problems such as those arising on the local memories of GPU accelerators. 
Table I. Model Parameters

\begin{tabular}{|c|c|}
\hline Constant & Latency \\
\hline \hline latency_local_memory & 8 \\
latency_main_memory & 128 \\
latency_move $\left(s_{v}\right)$ & $8+2 s_{v}$ \\
latency_spill $\left(s_{v}\right)$ & $128+4 s_{v}$ \\
latency_reload $\left(s_{v}\right)$ & $128+4 s_{v}$ \\
\hline
\end{tabular}

The results collected so far are very encouraging and indicate that the proposed approach competes with the best-fit, and may outperform it on the harder allocation problems with a small local memory size.

We start with the survey of our experimental methodology, then we provide an experimental evaluation of our heuristic and discuss the results.

\subsection{Methodology}

Our comparisons are based upon randomly generated graphs that attempt to reproduce the characteristics of real-life applications. We were inspired by the common characteristics found in representative numerical benchmarks, such as those we used in our previous work [Diouf et al. 2009] (BMCM [Berry et al. 1988], MXM, EDGE_DETECT [Lee 1998], and FFT [Lee 1998]) and the Polybench [Pouchet 2012]. These graphs have been used to evaluate our decoupled approach to the local memory allocation problem. ${ }^{3}$

Table I lists the parameters that model the local memory used in our experiments. We modeled a typical DDR memory with burst/pipelined and random-access latencies. Varying these ratios will change the overall benefits of local memory allocation, but not the relative performance of the different heuristics/algorithms.

Before we present the results of our evaluation, we first explain how we proceed to generate the random graphs, then we give a description of the compared algorithms, and finally we depict the details of our experimental evaluation.

8.1.1. Graph Generation. We generate interval graphs, selecting start (left) and end (right) points at random. We also try to reproduce the control and data structures found in real-life applications, adding specific constraints and limits on these graphs. We control the number of intervals, the minimum length of an interval, the maximum number of concurrently live intervals, and weight of intervals. Although there is no specific limit on maximum number of data structures that can be used in an application, in practice it is not usual to see hundreds of data structures in a single application. Also data structures in applications are usually not intended to be created and used at the same line of code. That is, when a data is created it is meant to be used for some time; they are created, used, and removed from memory when they are no longer required. By giving a bound on interval length we have ensured that the virtual data structures created within an application are used for a minimum length of time and the maximum number of live intervals within the graph is used to limit the maximum number of data elements concurrently used in an application.

Our graphs are composed of compound intervals which are in turn composed of basic intervals which have been introduced in Section 7. Each compound interval represents the whole live range of an array, and the basic intervals represent the subintervals of the compound interval where the array is frequently accessed. These basic intervals

\footnotetext{
${ }^{3}$ Extracting these graphs from real code would strengthen the experimental evaluation, assuming the extraction method implements loop transformations to improve data locality and to partition arrays into small, homogeneous blocks. Interference graphs may then be built from the live ranges of these array blocks [Diouf et al. 2009]. How to perform these steps automatically and profitably remains a largely open problem.
} 
Table II. Parameters for Graph Generation

\begin{tabular}{|c|c|}
\hline Parameter & Value \\
\hline \hline maximal loop nest & 4 \\
number of loops & 20 \\
minimal number of_outerloops & 5 \\
maximal number of outerloops & 5 \\
number of compound intervals & 30 \\
maximal number of basic intervals per compound interval & 3 \\
The number of different array's size & 10 \\
\hline
\end{tabular}

are called hot portions in the approach of Li et al. [2011]. Usually the array is accessed frequently through loops and in our implementation, each basic interval corresponds to an access to an array through a loop. A basic interval begins at the start point of a loop where an array is accessed and ends at the end point of that loop. Thus to generate the basic intervals, we first generate some loops that can be imbricated or not. Each loop has a start point and an end point that differs from start points and end points of other loops. A loop $l_{1}$, that contains a loop $l_{2}$, starts before $l_{2}$ and ends after $l_{2}$. For each array $A$, we randomly choose some loops where $A$ will be frequently accessed. A basic interval $b i$ of $A$ corresponds to a chosen loop $l$. The start point and the end point of $b i$ are set to the start point and end point of $l$. When choosing the basic intervals of $A$, when two basic intervals are such that the one is contained in the other, we consider that the $A$ is accessed in the containing basic interval. Each $b i$ is associated to a randomly generated frequency and is defined as being a write, meaning that $A$ is modified within $b i$, or a use, meaning that $A$ is only read within $b i$. After all the basic intervals of $A$ are chosen, we define $c i$, the compound interval of $A$ : it contains all the basic intervals of $A$, starts with the ourtermost loop containing the first basic interval of $A$, and ends with the outermost loop containing the last basic interval of $A$.

To make the generation of interval graphs as generic as possible we use the parameters presented in Table II. We were inspired by the frequent characteristics found in representative numerical benchmarks, such as the four aforementioned kernels and the Polybench [Pouchet 2012]. The parameters allow to control the number of intervals in the graph, the number of loops, the maximum depth of a loop, the maximum number of basic intervals of a compound interval, etc.

8.1.2. Algorithms. We use the randomly generated graphs to evaluate our approach by comparing it with three other approaches.

Classical best fit. This algorithm, denoted BestFit, walks over the list of basic intervals and attempts to assign every basic interval bi to a portion of the local memory (in fact, it is the array of this $b i$ which is assigned). If there is enough space to hold $b i$, it chooses the space where $b i$ fits the best. Otherwise either $b i$ is spilled or some previously assigned basic intervals are spilled to make room for $b i$.

Best-fit variant. This is a variant of of the best-fit, denoted BestFitVariant. Whenever a basic interval $b i$ ends, it is checked if $b i$ is the last basic interval of its compound interval $c i$. If so, $b i$ is removed from the local memory. Otherwise, $b i$ is left in the local memory until this space is needed for another basic interval $b i^{\prime}$ of another compound interval $c i^{\prime}$ (another array) or the next basic interval of $c i$ starts. The aim of this technique is to assign, if possible, different live ranges of an array to the same offset in the local memory in order to avoid copy costs.

Superperfect This is our implementation of the approach of $\mathrm{Li}$ et al. [2011] denoted here SuperPerfect. In this implementation the live range splitting is naturally performed because the frequently accessed portions (called hot portions by 


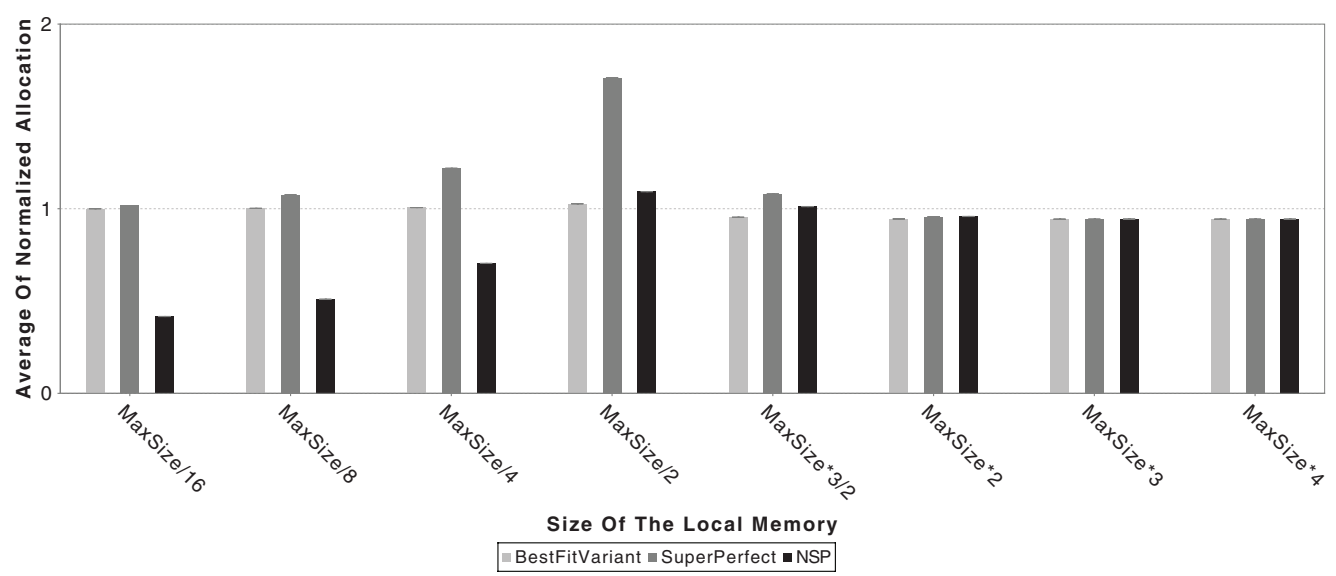

Fig. 7. Average of normalized allocation with respect to best-fit on superperfect graphs.

authors of the approach) of the array's live ranges are exactly the basic intervals. Thus, the set of candidates subject to allocation are composed of the compound intervals and their basic intervals. The allocation algorithm will consider either the compound interval or each of its individual basic intervals for allocation, but not both at the same time. To approximate a given graph into a superperfect graph, we go through the loops in their increasing start points, and we mark all the intervals (the candidates, the basic and compound intervals) defined at the same point as containing related; notice that two different loops have start points and end points that differ. When at a start point, an interval $j$ starts when another interval $i$ is already defined and still live, and $i$ and $j$ are not containing related, we extend $i$ to the end of $j$. After this approximation the new formed interval graph is superperfect.

8.1.3. Evaluation Details. Our evaluation was conducted on different local memory sizes. We varied the size of the local memory in accordance with MaxSize when performing the experiments; MaxSize-the maximum size of simultaneously living arrays-can vary significantly between two graphs. Thus, we do think that it is not very relevant to compare two allocation algorithms on graphs with MaxSize that widely differs when using a local memory with a fixed size. We focus here on the interesting case where MaxSize is the maximum size of simultaneously living basic intervals and not of the compound intervals, which is larger and thus makes the problem easier to solve.

We based our comparison on the cumulative memory access latency incurred by each method. The allocation of the best quality is the allocation with the lowest access latency. For every interval graph the cost of the allocation performed by BestFitVariant, SuperPerfect, and our approach, denoted NSP, have been normalized with respect to BestFit. For a given size of the local memory and a given algorithm, an associated bar shows the average (Figure 7 and Figure 9) and how the individual allocations are statistically distributed in the normalized allocation space (Figure 8 and Figure 10), of all the normalized allocation cost of the given algorithm.

To perform an allocation with our approach we feed Algorithm 3 with the list of basic intervals of each weighted interval graph. Algorithm 3 will then return a not-so-proper interval graph composed of local-memory live intervals. The MaxSize of the resulting graph is less than or equal to the size of the local memory, hence all its associated array blocks can be placed in the local memory using Algorithm 2. 


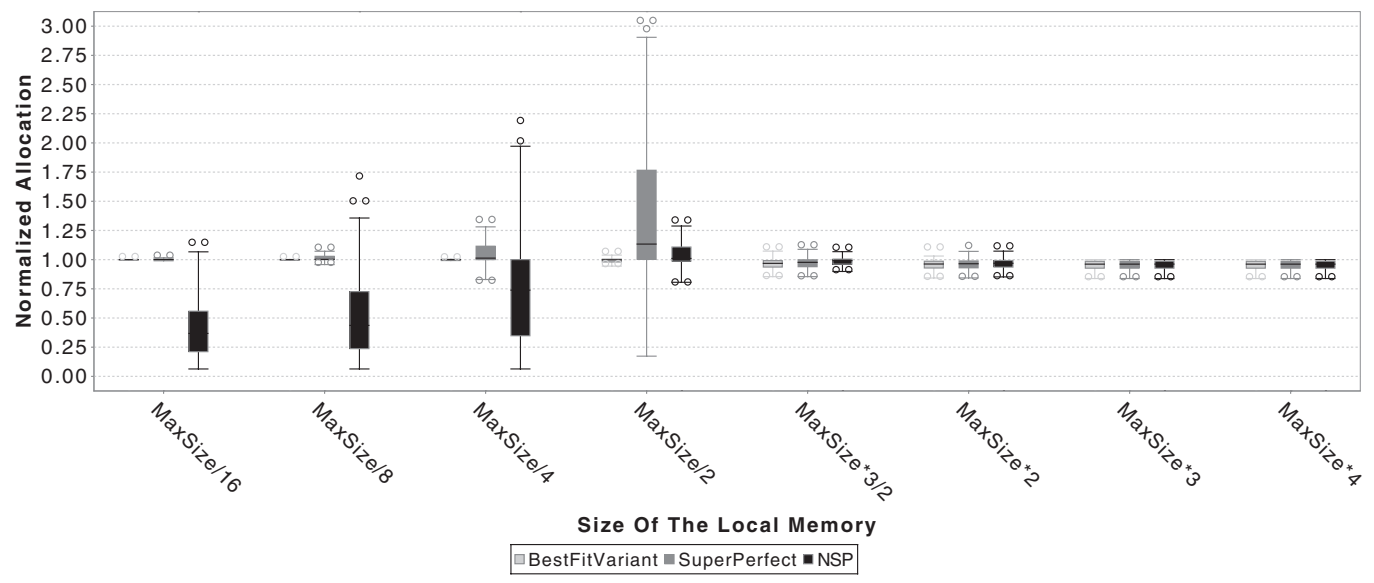

Fig. 8. Distribution of normalized allocation with respect to best-fit on superperfect graphs.

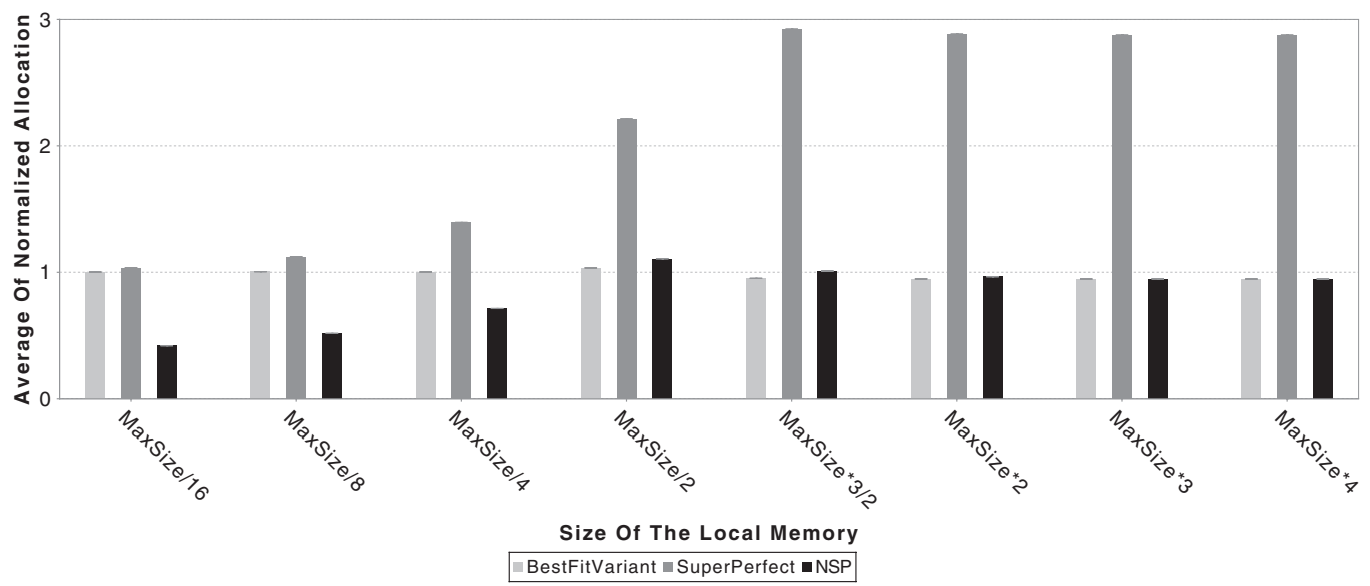

Fig. 9. Average of normalized allocation with respect to the best-fit on arbitrary graphs.

\subsection{Results and Discussion}

We provide here an evaluation of our approach, on 1000 of randomly generated superperfect graphs and 1000 of randomly generated arbitrary interval graphs. The results presented in this section show the performance of BestFitVariant variant (light gray bars), SuperPerfect allocator (gray bars), and our approach denoted NSP (black bars).

Figure 7 and Figure 8 present the results obtained on superperfect graphs. For all local memory sizes, our approach is better than SuperPerfect approach. Since the graphs are superperfect, there is no need of approximation for both approaches. Thus, the difference here is on the choice of allocated arrays. This shows that, on the generated graphs, our approach performs a better allocation. When the size of the local memory is lower or equal to MaxSize/4, our approach is better than BestFit and BestFitVariant. For local memory size of MaxSize/2, MaxSize, MaxSize $\times 3 / 2$, BestFit, and BestFitVariant give better results. For a size of the local memory going from MaxSize $\times 2$ to MaxSize $\times 4$, BestFitVariant, SuperPerfect, and NSP give similar results with small standard deviation. Algorithms produce approximately the same allocation because the size of the local memory is big enough to allow a good allocation. Since the 


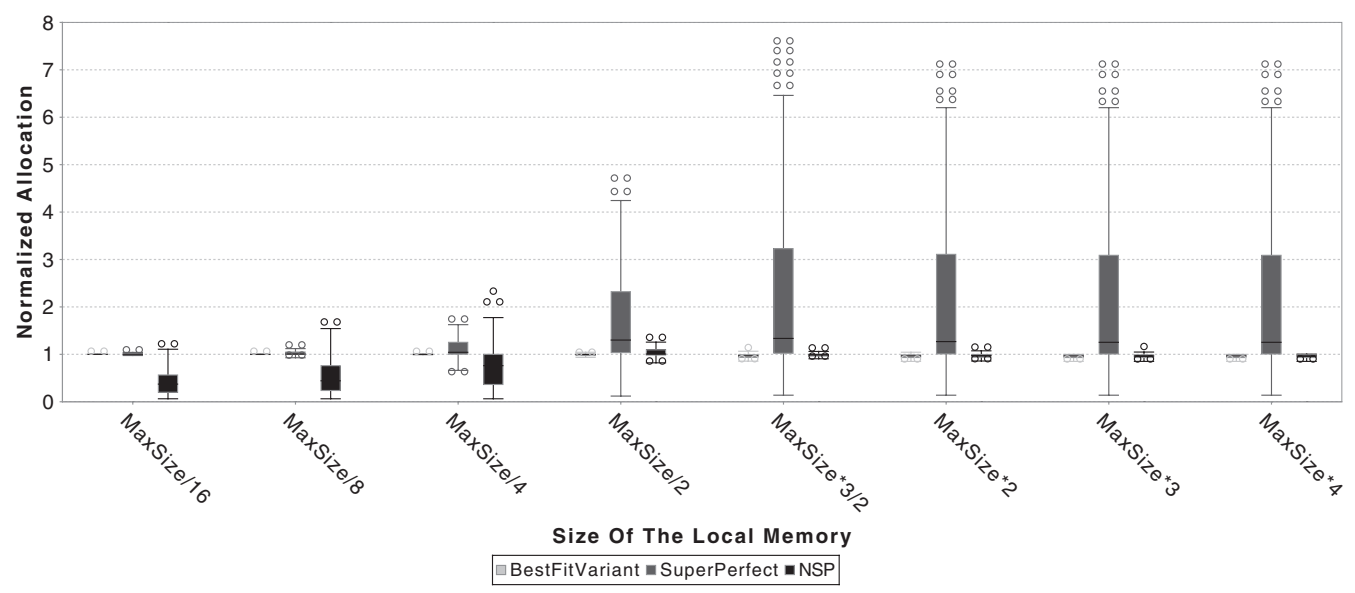

Fig. 10. Distribution of normalized allocation with respect to the best-fit on arbitrary graphs.

three algorithms try to assign to the basic intervals of a same array, the same place in the local memory, avoiding thus extra copies, they give better results compared to BestFit. But the small improvements suggest that these copies does not have a big impact on the global allocation cost.

Figure 9 and Figure 10 show the results obtained on arbitrary graphs. Here again, for all local memory sizes, our approach gives better results than SuperPerfect approach, and on arbitrary graphs. Our approach, like BestFit and BestFitVariant, performs on average allocations twice better than the allocations performed by SuperPerfect algorithm. This performance degradation, of the SuperPerfect algorithm, is mostly due to its approximation algorithm which is not meant to be used on graphs that are not quasi-superperfect, that are interval graphs that have most of their intervals matching the containment property. Comparing with BestFit and BestFitVariant, our approach gives similar results to those presented in Figure 8, but with a slightly higher variability.

\section{RELATED WORK}

Strong links between register allocation and local memory management have been discovered for more than 30 years by Fabri [1979]. Fabri's seminal paper also studied the interplay between local memory management and the loop transformations. Since then it has been ignored in the field of local memory management and register allocation [Appel and George 2001; Hack et al. 2006; Bouchez et al. 2006b; Quintáo Pereira and Palsberg 2008]. While previous studies addressed local memory management from different angles, targeting both code and data, we are especially interested in data management [Kandemir et al. 2001; Issenin et al. 2007; Dominguez et al. 2007]. We target dynamic methods which are superior to static ones except when code size is extremely constrained [Udayakumaran and Barua 2003]. We elaborate on two recent series of results targeting stack and global array management in local memories, embracing the analogies with register allocation. The first approach [Li et al. 2005] uses an existing graph coloring technique to perform memory allocation for arrays. It partitions the local memory for each array size, performs live range splitting, and uses a register allocation framework to perform memory coloring. The second approach [Avissar et al. 2002; Udayakumaran and Barua 2003; Udayakumaran et al. 2006] allocates data onto the scratchpad memory between program regions separated by specific program points. More specifically, allocation is based on the access frequency-per-byte 
of a variable in a region (collected from profile data). Program points are located at the beginning of a called procedure or before a loop entry.

The closest work to ours is $\mathrm{Li}$ et al. [2011], where authors observed that in many embedded applications most arrays present a specific live range behavior. Specifically, for any two arrays live ranges are either disjoint or one of the arrays is contained by the other one (containment property). They showed that, for the tested benchmarks, it is extremely rare to have two live ranges interfere with one another without containment. They extend the live range of one of the arrays to contain the other when this happens. Authors proved that the interference graph of an application with such a property is a comparability graph which is a superperfect graph and hence optimal interval coloring for this array interference graph is possible. Based on this observation, they rely on the maximum weighted clique to guarantee the optimal colorability of the generated interference graph. When the maximum weighted clique exceeds the size of the local memory, they use heuristics to spill or split some of the live ranges. While this work is interesting, it is restricted to applications where most arrays satisfy the containment property. On the other hand, our work leverages the decoupled allocation/assignment approach, allowing scalable and more effective algorithms. Moreover, it offers much more flexibility in terms of integration of architecture constraints and performance models.

\section{CONCLUSION}

We implemented a novel compilation-time local memory management approach through decoupling spill code generation and local memory assignment. We represent the live range intervals of variables and arrays as a weighted interval graph. We defined a new decision problem called the submarine-building problem, a variant of the ship-building problem. The submarine-building problem corresponds to coloring a weighted interval graph with a cyclic set of colors (corresponding to a wrap-around local memory). We demonstrate important complexity results on this problem, some of which are particularly original in graph theory. We provide a new clustering heuristic to approximate interval graphs into not-so-proper interval graphs, on which the submarine-building problem can be decided in linear time. This approximation effectively decouples the generation of spill code from the local memory assignment problem. Our preliminary experiments demonstrate the practicality of the approach, and very favorable allocation results compared to state-of-the-art allocators.

\section{REFERENCES}

Appel, A. W. AND George, L. 2001. Optimal spilling for CISC machines with few registers. In Proceedings of the ACM SIGPLAN Conference on Programming Language Design and Implementation (PLDI'01). 243-253.

ARM. 1998. Document no. arm ddi 0084d, ARM Ltd. ARM7TDMI-S data sheet.

Avissar, O., Barua, R., AND StewART, D. 2002. An optimal memory allocation scheme for scratch-pad-based embedded systems. ACM Trans. Embed. Comput. Syst. 1, 1, 6-26.

Berry, M., Chen, D., Koss, P., Kuck, D., Lo, S., Pang, Y., Pointer, L., Roloff, R., Sameh, A., Clementi, E., Chin, S., Schneider, D., Fox, G., Messina, P., Walker, D., Hsiung, C., Schwarzmeier, J., Lue, K., Orszag, S., Seidl, F., Johnson, O., ANd Goodrum, R. 1988. The perfect club benchmarks: Effective performance evaluation of supercomputers. Int. J. Supercomput. Appl. 3, 5-40.

Boissinot, B., Darte, A., de Dinechin, B. D., Guillon, C., and Rastello, F. 2009. Revisiting out-of-ssa translation for correctness, code quality and efficiency. In Proceedings of the Annual IEEE/ACM International Symposium on Code Generation and Optimization (CGO'09). 114-125.

Bouchez, F., Darte, A., Guillon, C., and Rastello, F. 2006a. Register allocation: What does the npcompleteness proof of Chaitin et al. really prove? In Proceedings of the Annual Workshop on Duplicating, Deconstructing and Debugging (WDDD'06).

Bouchez, F., Darte, A., Guillon, C., AND Rastello, F. 2006b. Register allocation: What does the npcompleteness proof of Chaitin et al. really prove? Or revisiting register allocation: Why and how. 
In Proceedings of the International Workshop on Languages and Compilers for Parallel Computing (LCPC'06). Lecture Notes in Computer Science. Springer.

Bouchez, F., Darte, A., And Rastello, F. 2007. On the complexity of register coalescing. In Proceedings of the Annual IEEE/ACM International Symposium on Code Generation and Optimization (CGO'07).

Bouchez, F., Darte, A., and Rastello, F. 2008. Advanced conservative and optimistic register coalescing. In Proceedings of the International Conference on Compilers Architecture and Synthesis for Embedded Systems (CASES'08). 147-156.

BRAUn, M. AND HACK, S. 2009. Register spilling and live-range splitting for ssa-form programs. In Proceedings of the International Conference on Compiler Construction (CC'09). Lecture Notes in Computer Science, vol. 5501. Springer, 174-189.

Brisk, P., DABiRi, F., JAfARI, R., AND SARRAFZAdeH, M. 2006. Optimal register sharing for high-level synthesis of ssa form programs. IEEE Trans. Comput. Aid. Des. Integr. Circ. Syst. 25, 5, 772-779.

Burns, M., Prier, G., Mirkovic, J., And Reiher, P. 2003. Implementing address assurance in the intel ixp. Western Network Processors Conference.

Chen, L. 1992. Optimal parallel time bounds for the maximum clique problem on intervals. Inf. Process. Lett. 42, 4, 197-201.

Diouf, B., Ozturk, O., AND Cohen, A. 2009. Optimizing local memory allocation and assignment through a decoupled approach. In Proceedings of the $22^{\text {nd }}$ International Workshop on Languages and Compilers for Parallel Computing (LCPC'09).

Dominguez, A., NGuYen, N., AND Barua, R. K. 2007. Recursive function data allocation to scratch-pad memory. In Proceedings of the International Conference on Compilers Architecture and Synthesis for Embedded Systems (CASES'07). 65-74.

FABRI, J. 1979. Automatic storage optimization. In Proceedings of the ACM Symposium on Compiler Construction. 83-91.

Golumbic, M. C. 2004. Algorithmic graph theory and perfect graphs. Ann. Discr. Math. 57.

Hack, S., Grund, D., ANd Goos, G. 2005. Towards register allocation for programs in ssa-form. Tech. rep. 2005-27, Universität Karlsruhe.

Hack, S., Grund, D., AND Goos, G. 2006. Register allocation for programs in ssa-form. In Proceedings of the ACM Symposium on Compiler Construction (CC'06). 247-262.

Instruments, T. 1997. TMS370Cx7x 8-Bit Microcontroller. Texas Instruments.

Issenin, I., Brockmeyer, E., Miranda, M., And Dutt, N. 2007. DRDU: A data reuse analysis technique for efficient scratch-pad memory management. ACM Trans. Des. Autom. Electron. Syst. 12, 2, 15.

Kahle, J. A., Day, M. N., Hofstee, H. P., Johns, C. R., Maeurer, T. R., And Shippy, D. 2005. Introduction to the cell multiprocessor. IBM J. Res. Devel. 49, 4-5.

Kandemir, M., Ramanujam, J., Irwin, J., Vijaykrishnan, N., Kadayif, I., and Parikh, A. 2001. Dynamic management of scratch-pad memory space. In Proceedings of the Design Automation Conference (DAC'01). 690-695.

LEE, C. G. 1998. UTDSP benchmarks. http://www.eecg.toronto.edu/ corinna/DSP/infrastructure/UTDSP.html

Lee, J. K., Palsberg, J., AND Pereira, F. M. Q. 2008. Aliased register allocation for straight-line programs is np-complete. Theor. Comput. Sci. 407, 258-273.

Li, L., FEnG, H., AND XuE, J. 2009. Compiler-Directed scratchpad memory management via graph coloring. ACM Trans. Archit. Code Optim. 6, 9:1-9:17.

LI, L., GAO, L., AND XUE, J. 2005. Memory coloring: A compiler approach for scratchpad memory management. In Proceedings of the International Conference on Parallel Architecture and Compilation Techniques (PACT'05). 329-338.

Li, L., XUE, J., AND KNOOP, J. 2011. Scratchpad memory allocation for data aggregates via interval coloring in superperfect graphs. ACM Trans. Embed. Comput. Syst. 10, 28:1-28:42.

Motorola. 1998. M-CORE: MMC2001 Reference Manual. Motorola Corporation.

NVIDIA. 2008. NVIDIA unified architecture GeForce 8800 GT. http://www.nvidia.com/page/geforce8.html.

Pereira, F. And Palsberg, J. 2009. Ssa elimination after register allocation. In Proceedings of the ACM Symposium on Compiler Construction (CC'09). O. Moor and M. Schwarzbach, Eds. Lecture Notes in Computer Science, vol. 5501. Springer, 158-173.

Pereira, F. M. Q. And Palsberg, J. 2005. Register allocation via coloring of chordal graphs. In Proceedings of the Asian Symposium on Programming Languages and Systems (ASPLAS'05). 315-329.

Pereira, Q. F. M. ANd PALsberg, J. 2008. Register allocation by puzzle solving. SIGPLAN Not. 43, 6, $216-226$.

Pouchet, L. N. 2012. Polybench/c, the polyhedral benchmark suite. http://www.cse.ohio-state. edu/ pouchet/software/polybench/ 
Saha, A., Pal, M., and Pal, T. K. 2007. Selection of programme slots of television channels for giving advertisement: A graph theoretic approach. Inf. Sci. 177, 12, 2480-2492.

SARKAR, V. AND BARIK, R. 2007. Extended linear scan: An alternate foundation for global register allocation. In Proceedings of the ACM Symposium on Compiler Construction (CC'07). S. Krishamurthi and M. Odersky, Eds. Lecture Notes in Computer Science, vol. 4420. Springer, 141-155.

SJodin, J. and von Platen, C. 2001. Storage allocation for embedded processors. In Proceedings of the International Conference on Compilers, Architecture and Synthesis for Embedded Systems (CASES'01). ACM Press, New York, 15-23.

Steinke, S., WehmeYer, L., Lee, B., ANd Marwedel, P. 2002. Assigning program and data objects to scratchpad for energy reduction. In Proceedings of the Conference on Design, Automation and Test in Europe (DATE'02). IEEE Computer Society, Washington, DC, 409.

UdAYAKUMARAn, S. AND BARUA, R. 2003. Compiler-Decided dynamic memory allocation for scratch-pad based embedded systems. In Proceedings of the International Conference on Compilers Architecture and Synthesis for Embedded Systems (CASES'03). 276-286.

UdaYakumaran, S., Dominguez, A., AND Barua, R. 2006. Dynamic allocation for scratch-pad memory using compile-time decisions. ACM Trans. Embed. Comput. Syst. 5, 2, 472-511.

Received June 2012; revised September 2012; accepted October 2012 